\title{
The use of curcumin in modeling gerontological drinks with enhanced flavor
}

\author{
Nataliya Zavorokhina, Olga Chugunova*, and Olga Feofilaktova \\ Ural State University of Economics, 62/8, 8 Marta/Narodnoy Voli street, Yekaterinburg, Russia, \\ 620144
}

\begin{abstract}
In connection with the emerging global trend of an increase in the number of elderly people, an increase in morbidity and age-related changes occurring in the body, including a deterioration in taste and olfactory sensitivity, there is a need to provide rational nutrition for this category of citizens. The aim of the study was to model the formulations of gerontological drinks with enhanced flavor with a low glycemic index, with enhanced functional properties and containing the steviosidecurcumin complex. The study was carried out in the city of Yekaterinburg in three stages: investigated the taste and olfactory sensitivity in people aged 25-40 years and 65-90 years, investigated the effect of stevia extracts on the solubility of curcumin, simulated samples of drinks. It was found that the recognition of tastes in people in the age group of 65-90 years is worse in comparison with people in the age group of 25-40 years: for salty - by $37.8 \%$, for sour - by $39.0 \%$, for bitter - by $64,0 \%$, for sweets by $6.0 \%$. Olfactory sensitivity is $25.9 \%$ worse. Disease sensitivity is significantly affected by the disease: sensitivity is worse in diseases of the digestive system by $22.9 \%$, circulatory system - by $26.8 \%$, endocrine system - by $25.2 \%$, genitourinary system - by $26.1 \%$. To enhance the flavor intensity, yeast extract and the stevioside-curcumin complex obtained by extraction of curcumin from turmeric rhizome powder with long ethyl alcohol using stevioside were added to the drinks. It was found that with the addition of stevioside in an amount of $8 \%$, the maximum yield of curcumin was observed $-14.33 \pm 0.3 \mathrm{mg} / \mathrm{ml}$. Then they developed model samples of drinks, the sweetness profile of which is close to that of sugar, the aftertaste is 3 times longer, the taste intensity is $15 \%$ higher, antioxidant activity is 5.7 times more than that of a competitor.
\end{abstract}

\section{Introduction}

The demographic situation in the world in recent decades is characterized by an increase in the number of people over working age in the general structure of the population $[1,2]$. According to the results of 2017, the share of the population of this age group in Russia amounted to $25.4 \%$, according to the average version of the UN forecast by 2050 , the indicator will reach $31.2 \%$. Now every fourth resident is an old-age pensioner. In addition, there is a tendency towards an increase in average life expectancy: in 2017, in Japan, the

\footnotetext{
*Corresponding author: chugunova@usue.ru
} 
USA, and EU countries, it exceeded 80 years, in Russia it reached a historical maximum and amounted to 72.5 years. These demographic processes lead to a significant increase in the coefficient of the demographic burden on society [3]. As a result, people over working age are forced to increase their professional ability to work and productivity. However, due to age-related changes, under the influence of environmental factors, the state of health worsens, working capacity decreases, and, consequently, labor productivity, which affects the economic development of the state.

By reducing the number of acute diseases and increasing diseases associated with the progression of chronic pathological processes, the structure of morbidity significantly changes in old age, and mortality from external causes and infectious diseases decreases. The most common classes of causes of death in Russia in 2016: circulatory system diseases - $67.2 \%$, neoplasms $-19.4 \%$, digestive diseases $-4.8 \%$, respiratory diseases $-4.3 \%$, external causes $-3,9 \%$, some infectious and parasitic diseases - $0.4 \%$ [4]. Compared to people of working age, the mortality rate of the elderly population from diseases of the circulatory system is 2.1 times higher and from neoplasms, 1.15 times higher. A serious problem is an increase in the frequency of chronic neurodegenerative diseases (primarily Alzheimer's and Parkinson's diseases), for which today there are no radical methods of treatment to stop the pathological process [5]. Therefore, it is necessary to develop measures aimed at improving the quality of life, preventing diseases, and maintaining the health of the population.

The most important factor affecting health status and longevity is nutrition. In this regard, interest in gerontodietology is growing - a science that studies the nutrition problems of elderly and senile people, as well as people with a high risk of early and pathological aging, aimed at developing scientifically based recommendations regarding rational, preventive and therapeutic nutrition, methods of its organization for this group of population.

The problem of rational, balanced, nutritionally adequate age is reflected in a number of regulatory documents and legislative acts of the Russian Federation. In 2010, by Order of the Government of the Russian Federation of October 25, $2010 \mathrm{~N}$ 1873-o. The "Fundamentals of the state policy of the Russian Federation in the field of healthy nutrition for the period up to 2020" were approved. By the order of the Government of the Russian Federation of February 5, 2016 N 164-o. The document "Strategy for the interests of senior citizens in the Russian Federation until 2025" was approved, which confirms the relevance of the question of creating a list of special multicomponent food products for elderly citizens, developed taking into account the physiological norms of energy and nutrient requirements. When developing such products, it is necessary to take into account the fact that olfactory and taste sensitivity worsens with age. Therefore, for older people, the flavor of the products becomes insufficiently saturated, a decrease in appetite is observed, and, as a result, nutritional deficiency, the functioning of all body systems is disrupted [6].

Methven L., Allen VJ, Withers CA found that with age, the intensity of taste decreases to all four tastes (sour, salty, bitter, sweet), however, the loss of sensitivity varies depending on taste: for salty and sour - the decrease reaches $80 \%$, for sweet and bitter - 70\% [7], Solemdal K., Sandvik TL in their studies report a greater decrease in sensitivity to sour and bitter tastes [8]. The reason for the inconsistency of the results was the use of different solutions of flavoring substances: to identify the acidic taste - citric or acetic acids. Perception of salty taste worsens with diseases of the circulatory system [9]. Taste can not only deteriorate, but also pervert. Elderly people complain of bad taste in the mouth, discomfort, or even a complete lack of any taste.

Olfactory function is disturbed mainly in long-livers and senile people. This phenomenon is called senile hyposmia. Chen J. and Rosenthal A. found that more than $50 \%$ of elderly people aged 65 to 80 years and $75 \%$ older than 80 years experience significant olfactory disorders [10]. Bukreyev N.S. claims that in addition to this, older people get used 
to smells faster and restore sensitivity more slowly than young people [11]. Lafreniere D., Mann N. confirmed that the sense of smell affects the perception of tastes $(2 / 3$ of the ability to distinguish the taste of food is taken by the olfactory ability), but this effect is not taken into account in many studies [12]. Thus, the inconsistency of the results of many studies in this area requires a more detailed study of the issue of reducing taste and olfactory sensitivity with age.

Since with age a significant deterioration in the taste and olfactory sensitivity of a person is noted due to disturbances in the functioning of the sensory apparatus associated with the appearance of chronic diseases and other factors, when modeling the composition of gerontological drinks, it is necessary to use ingredients that have an intense flavor or enhance it.

Curcuminoids can become promising prescription components of new gerontological drinks. Curcuminoids are the main group of biologically active compounds contained in the rhizome of the turmeric plant (2-9\%, depending on the place of growth). The dominant compounds are curcumin, desmethoxycurcumin and bisdemethoxycurcumin. They determine the yellow-orange color of the raw material and determine the pharmacological spectrum of plant preparations. Turmeric (Curcuma longa) is a perennial herb in the ginger family (Zingiberaceae). Turmeric rhizome is used as a spice, food coloring and medicinal plant. One of the main ingredients in Indian curry seasoning. It grows in tropical countries in India, Iran, Indonesia, China, Vietnam, and has been cultivated in Azerbaijan since 1986. The plant is popular in modern traditional medicine of the Central Asian countries

The main curcuminoid is curcumin, its content reaches $0.6 \%$. Curcumin is a lipophilic bioflavonoid (diferuloylmethane) insoluble in water and soluble in alkalis and in most organic solvents. To extract curcumin from natural sources, researchers use organic solvents such as acetone, methanol, ethanol, ethyl acetate, isopropanol and hexane, using coprecipitation, dipping, ultrasound, microwave processing and subsequent crystallization [13]. Curcumin is associated with antioxidant, anti-inflammatory, anticoagulant, anticholesterolemic, antibacterial, antidepressant, hypoglycemic, immunomodulating, antimutagenic, anti-amyloid properties [14, 15].

Curcumin, participating in the processes of neurogenesis, carcinogenesis, helps to prevent neurodegenerative diseases, cancer. With antioxidant properties, it prevents the occurrence of diseases of the circulatory system, including those caused by aging. Despite the therapeutic potential, safety, even at high doses of up to 8-12 g per day, the use of curcumin is difficult in scientific medicine and the food industry due to its poor solubility in water and low bioavailability.

V. C. Sezgin, O. Bayraktar from the USA to increase the solubility, bioavailability received a complex compound of curcumin with piperine - extract of black pepper, which is able to increase the bioavailability of curcumin by 20 times (Pat. EP3142702B1 US) [16]. However, the resulting compound causes a burning sensation, which makes it impossible to use it for the preparation of drinks.

R. Jager, R. P. Lowery developed a compound of curcumin with a hydrophilic carrier called "CurcuWin." According to a study, CurcuWin increased levels of curcuminoids in blood plasma by 46 times, compared with standard curcumin, which indicates its high bioavailability [17].

To increase the solubility of curcumin in water, nanoparticles, liposomes, micelles, and phospholipid complexes [18] rebaudioside [19] were used. The creation of liposomal forms of curcumin can increase its bioavailability up to 50\% compared with the native form. However, liposomes are unstable during storage and also with respect to the environment in the stomach.

The brand Merviva has patented a combination of curcumin with phospholipids (phosphatidylcholines) of soya lecithin (US Pat. WO2013176555A1 US), which are 
surface-active compounds and are able to dissolve in both water and oil, $450 \mathrm{mg}$ of the resulting drug is equivalent to $4000 \mathrm{mg}$ of curcumin [20 ].

D. Benam developed the micellar form of curcumin (Pat. RU2615815C2 RF), on the basis of which a bioavailability of at least 230 times increased compared to native curcumin was revealed. The formulation contains curcumin, emulsifiers Polysorbate 80 , Polysorbate 20 , or a mixture thereof [21]. The disadvantage of solubilizates obtained using these emulsifiers is their possible toxicity to normal and malignant cells [22].

O. A. Legonkova, A. I. Korotaeva developed a method for producing bioavailable curcumin by mixing curcumin with an alcohol colloidal solution of nanocrystalline cerium dioxide stabilized with polyvinylpyrrolidone and then drying the resulting colloidal solution (Pat. RU2665378C1 RF) [22]. The resulting composite has a reduced cytotoxicity and increased bioavailability.

Although there are many ways to obtain water-soluble curcumin compounds, they are all complex and expensive. The simplest preparation of water-soluble curcuminoids is the method using stevia extracts developed by H. Nguyen, J-B Si, C. Kang [23]. However, the authors have not fully developed the technological process for obtaining a complex of curum with stevia extracts, the ratio of prescription components to ensure acceptable organoleptic characteristics and functional properties is not determined, the finished product yield is not presented, the method for determining the mass fraction of curcumin in the compound with stevia extracts is not indicated. The practical application of the research results is not presented. This circumstance is important because the resulting complex of curcumin with stevia extracts has a very sweet taste, which makes it difficult to use. It is necessary to determine the sweetness of the compound compared to sugar and, based on the recommended daily requirement for curcumin, suggest options for its use. These shortcomings allow further study of this issue.

Based on the foregoing, the main research problem is formulated - the problem of expanding the assortment of gerontological products with enhanced flavor with predetermined properties, containing scarce macro- and micronutrients and intended for the prevention of nutrition-related diseases and sensory disturbances. Drinks are a convenient and affordable form of obtaining nutrients necessary for the correction of nutritional status by introducing ingredients that have therapeutic and prophylactic properties. Therefore, the aim of the study is to model the formulations of gerontological drinks with enhanced flavor with a low glycemic index, which have enhanced functional properties and contain the stevioside-curcumin complex.

The hypothesis of the study is the assumption that it is possible to obtain and use a water-soluble curcumin compound with increased bioavailability in modeling the formulations of gerontological drinks with enhanced functional properties and a more intense taste and intense aroma for elderly people.

To achieve this goal, three main tasks were solved:

- to obtain quantitative values in relation to taste and olfactory sensitivity of persons in two age categories: $25-40$ years and 65 years and older;

- study the effect of stevia extracts (stevioside and rebaudioside) on the solubility of curcumin;

- to simulate a gerontological drink taking into account the obtained results.

\section{Materials and methods}

Research methods of taste and olfactory sensitivity. Objects: samples of solutions of the main flavoring substances (sour, salty, bitter, sweet), olfactory samples.

Experimental studies were carried out at the Ural State University of Economics (USUE) in the laboratories of the Department of Food Technology and with the assistance 
of the Council of Veterans of the USUE. At the first stage, the taste and olfactory sensitivity of persons aged 25-40 years was studied in comparison with persons aged 65-90 years. All respondents lived in the city of Yekaterinburg.

According to the data of the "Office of the Federal State Statistics Service for the Sverdlovsk Region," the distribution of the population of the city of Yekaterinburg by sex and age was established, thereby determining the general population: men and women aged 25-40 years amounted to 345.7 thousand people, men - 165.1 thousand people or $47.8 \%$, women - 180.1 thousand people or 52.2\%; men and women aged $65-90$ years amounted to 161.1 thousand people, men - 49.3 thousand people or $30.6 \%$, women -112.3 thousand people or $69.4 \%$.

Next, the size of the sample is determined. The number of sampling units should be directly proportional to the number of such units in the population. Confidence probability $95 \%$, confidence interval (error) $\pm 5 \%$. The sample size of respondents aged 25-40 years (average age $32.6 \pm 5.3$ years) was 384 people, 184 men, 200 women; at the age of 65-90 years (64 men, 143 women) - 383 people, 117 men, 266 women.

The study of taste sensitivity to basic tastes (sour, salty, sweet, bitter) was carried out in accordance with GOST ISO 3972-2014. Closed tastings were held, samples were encoded with a three-digit digital code. Coded samples for each respondent and additional materials were placed on the tables: tasting sheets, pens or pencils, non-carbonated drinking water for rinsing the mouth between sample samples and restoring receptor sensitivity. During the tests, they were guided by the requirements described in GOST ISO 3972-2014.

In preparing the experiment, samples were prepared as follows. First, basic solutions were prepared using reference food-grade chemicals, table 1.

Table 1. Test Solution Specification

\begin{tabular}{|l|l|l|}
\hline Taste & Reference substance & Concentration, g / dm3 \\
\hline Sour & Citric Acid, monohydrate & 1,2 \\
\hline Salty & Sodium Chloride, anhydrous & 4,0 \\
\hline Sweet & Chemically pure sucrose & 24,0 \\
\hline Bitter & Caffeine, monohydrate & 0,54 \\
\hline
\end{tabular}

A series of solutions were prepared from the main solutions. According to each taste, samples were prepared in two concentrations of Id and D5 g / dm3, respectively: acidic (citric acid monohydrate) 0.28 and 0.48 ; salted (sodium chloride anhydrous) -1.19 and 0.48 ; sweet (chemically pure sucrose) - 5.76 and 1.56; bitter (caffeine monohydrate) -0.195 and 0.11 . The rationale for choosing these concentrations is that, based on practical tests, it has been proven that more than $50 \%$ of novice testers have recognized a large concentration of Id.

Tests were performed without haste with an interval between each test of approximately 30 seconds. The amount of sample of each sample is not less than $15 \mathrm{~cm} 3$. Respondents were offered 10 samples of flavoring solutions. Each taste was presented twice (two samples of sour taste, two samples of salty taste, two samples of bitter taste, two samples of water). The number of samples corresponded to the number of respondents. Before carrying out the test, an explanatory conversation was held with the respondents about the tasting rules, the procedure for determining tastes was established, and it was verified that the respondents understood the task. The respondent consistently tested 10 samples of samples and recorded in the tasting sheet which of the five tastes he felt. Repeated sampling was not permitted. The results were described as follows: the sensation is not perceived - 0 , the sensation is perceived, but not identified -?, The taste is felt - the name of the taste (sour, sweet, bitter, salty, water).

In order to investigate olfactory sensitivity, we used the methodology presented in GOST ISO 5496-2014. The olfactory assessment was performed by the direct method by evaluating olfactory samples. Respondents were offered olfactory samples containing 
different odors of a given concentration. A two-digit recognition code was applied to vials and corks.

Respondents were asked to recognize 10 aqueous solutions, the most common odors in everyday life, table 2 .

Table 2. List of odors or odors for olfactory sensitivity assessment

\begin{tabular}{|c|c|c|c|}
\hline Name & Formula & Description & Concentration, g / dm3 \\
\hline Butyric acid & $\mathrm{C} 4 \mathrm{H} 8 \mathrm{O} 2$ & Cheesy & \multirow{2}{*}{$10^{-3}$} \\
\hline Citral (geraneal and neral) & $\mathrm{C} 10 \mathrm{H} 16 \mathrm{O}$ & Lemon & \\
\hline Methionol & $\mathrm{C} 4 \mathrm{H} 8 \mathrm{OS}$ & Fried onion & \multirow{2}{*}{$5 \cdot 10^{-4}$} \\
\hline Benzyl acetate & $\mathrm{C} 9 \mathrm{H} 10 \mathrm{O} 2$ & Floral & \multirow{2}{*}{$5 \cdot 10^{-3}$} \\
\hline Terpinyl acetate & $\mathrm{C} 12 \mathrm{H} 20 \mathrm{O} 2$ & Coniferous & \\
\hline Ethylbutanate & $\mathrm{C} 6 \mathrm{H} 12 \mathrm{O} 2$ & Banana & \\
\hline cis-3-Hexen-1-ol & $\mathrm{C} 6 \mathrm{H} 12 \mathrm{O}$ & Grass & \\
\hline Benzaldehyde & $\mathrm{C} 7 \mathrm{H} 6 \mathrm{O}$ & Almond & \\
\hline$\gamma$-undecalactone & $\mathrm{C} 11 \mathrm{H} 20 \mathrm{O} 2$ & Fruity & \\
\hline Anethol & $\mathrm{C} 10 \mathrm{H} 12 \mathrm{O}$ & Anise & \\
\hline
\end{tabular}

To study olfactory sensitivity, chemicals and compounds were used that correspond to the aroma: cheese - butyric acid, lemon - citral, fried onions - methionol, floral - benzyl acetate, coniferous - terpilacetate, grass - cis-3-Hexene-1-ol, almonds - benzaldehyde, fruit - $\gamma$-undecalactone, anise - anethole.

Research methods for the effects of stevia extracts (stevioside and rebaudioside) on the solubility of curcumin. Objects of study: ground turmeric "Aidigo", rectified ethyl alcohol of the highest grade according to GOST 18300 ("Extra-M" brand, "Belkhim" OJSC), stevia extracts with different degrees of purification "PureCircle" - "SWETA" stevioside, "REBAUDIOSIDE A rebaudioside 97\%" (Reb-A 97\%), the obtained complexes of stevioside-curcumin, rebaudioside-curcumin.

The list of components required to obtain complexes of stevioside-curcumin, rebaudioside-curcumin is presented in table 3.

Table 3. List of required components

\begin{tabular}{|c|c|c|c|c|c|c|}
\hline \multirow{2}{*}{ Component Name } & \multicolumn{5}{|c|}{ Mass fraction of stevia extracts } & \multirow{2}{*}{ Control } \\
\cline { 2 - 5 } & $2 \%$ & $4 \%$ & $6 \%$ & $8 \%$ & $10 \%$ & \\
\hline Curcuma powder, g & 1,0 & 1,0 & 1,0 & 1,0 & 1,0 & 1,0 \\
\hline Stevioside or rebaudioside, g & 0,2 & 0,4 & 0,6 & 0,8 & 1,0 & - \\
\hline Ethyl alcohol, cm3 & 6,8 & 6,6 & 6,4 & 6,2 & 6,0 & 7,0 \\
\hline Water, cm3 & 2,0 & 2,0 & 2,0 & 2,0 & 2,0 & 2,0 \\
\hline Total & 10,0 & 10,0 & 10,0 & 10,0 & 10,0 & 10,0 \\
\hline
\end{tabular}

Sample preparation: Stevia extracts (mass fraction from 2 to $10 \%$ ), $2 \mathrm{~cm} 3$ of water are added to the test tube, mixed until dissolved, turmeric powder is added in an amount of $1,000 \mathrm{~g}$, ethyl alcohol is added to a volume of $10 \mathrm{~cm} 3$, and mixed. The resulting mixture was shaken for 15 minutes and centrifuged at $1000 \mathrm{rpm}$. within $10 \mathrm{~min}$.

For centrifuging samples, a desktop laboratory centrifuge SM-12 was used. The supernatant is filtered through a "white ribbon" filter, pore size 5-8 microns. The alcohol is evaporated, the obtained powders are dissolved in water, centrifuged at $1000 \mathrm{rpm}$. for 10 min, filtered, then quantitatively determine curcumin in solution.

The mass fraction of curcumin was determined by the photocolorimetric method according to GOST 34146-2017 using a KFK-2 photocolorimeter with a measurement range in the wavelength interval from $315-980 \mathrm{~nm}$, with an admissible absolute transmittance error of not more than $+1 \%$. The method is based on determining the mass fraction of curcumin by measuring the color intensity of its solution. In contrast to the 
proposed method, distilled water rather than ethyl alcohol was used as a solvent, since the resulting compound was well soluble in water.

To prepare a solution of the the analyzed substance in a glass with a capacity of $50 \mathrm{~cm} 3$ is placed $0,0800 \mathrm{~g}$ of this substance and $20 \mathrm{~cm} 3$ of water, mix the contents with a glass rod until completely dissolved. Then the solution is transferred quantitatively to a volumetric flask (with a ground stopper) with a capacity of $200 \mathrm{~cm} 3$, the volume is brought to the mark with water, closed with a stopper and stirred. In a volumetric flask (with a ground stopper) with a capacity of $100 \mathrm{~cm} 3$, add $1 \mathrm{~cm} 3$ of the resulting solution with a pipette and bring the volume to the mark with water, close the stopper and mix. The photocolorimeter cuvette is filled with the prepared solution and the optical density is measured at a wavelength corresponding to the maximum light absorption $(425+1) \mathrm{nm}$ relative to the optical density of distilled water.

Mass fraction of curcumin in the analyzed compound X,\%, calculated by the formula:

$$
X=\frac{A \cdot \rho \cdot V \cdot V_{2}}{E_{1 c m}^{1 \%} \cdot d \cdot m \cdot V_{1}}
$$

where $\mathrm{A}$ is the optical density of the solution of the analyzed substance, OD units; $\rho$ is the density of the analyzed substance, numerically equal to the density of distilled water, 1 $\mathrm{g} / \mathrm{cm} 3$; $\mathrm{V}$ is the volume of the analyzed substance for the first dilution, $200 \mathrm{~cm} 3$; V2 is the volume of the analyzed substance for the second dilution, $100 \mathrm{~cm} 3$; - specific light absorption coefficient, numerically equal to the optical density of the solution of the analyzed substance, with a mass fraction of $1 \%$ with an absorbing layer thickness of $1 \mathrm{~cm}$, 1607 OD units $\%-1 \cdot \mathrm{cm}-1$; $\mathrm{d}$ is the thickness of the absorbing layer, $1 \mathrm{~cm} ; \mathrm{m}$ is the mass of the sample taken for analysis, $0,0800 \mathrm{~g}$; V1 is the volume of a solution of the analyzed substance taken for dilution, $1 \mathrm{~cm} 3$.

Statistical processing of research results. The absolute value of the difference between the results of two parallel determinations obtained when determining the mass fraction of curcumin, under repeatability conditions with a confidence probability of $\mathrm{P}=95 \%$, does not exceed the repeatability limit $r$ according to the table. The absolute value of the difference between the results of two parallel determinations obtained under reproducibility conditions with a confidence probability of $\mathrm{P}=95 \%$ does not exceed the reproducibility limit R. The boundaries of the absolute error in determining the mass fraction of the main dye in the dye A to be analyzed at $\mathrm{P}=95 \%$ are presented in table 4 .

Table 4. Boundaries of absolute error

\begin{tabular}{|c|c|c|}
\hline $\begin{array}{c}\text { Repeatability g,\% abs., At P } \\
=95 \%, \mathrm{n}=2\end{array}$ & $\begin{array}{c}\text { Reproducibility limit } \mathrm{R}, \% \\
\text { abs., At } \mathrm{P}=95 \%, \mathrm{~m}=2\end{array}$ & $\begin{array}{c}\text { The boundaries of the absolute } \\
\text { error } \Delta, \\
\%, \text { at } \mathrm{P}=95 \%\end{array}$ \\
\hline 0,40 & 0,80 & $\pm 0,4$ \\
\hline
\end{tabular}

\section{Results}

The result of determining the mass fraction of curcumin is represented as $(\mathrm{X} \pm \Delta) \%$, at $\mathrm{P}=$ 95\%.

Analysis of variance was performed using STATISTICA and Excel. The standard error of the mean of three single measurements is determined. The significance level is $5 \%$.

The results of the study of taste and olfactory sensitivity. The results of recognition by respondents of the main tastes in different age categories are presented in Figure 1. 


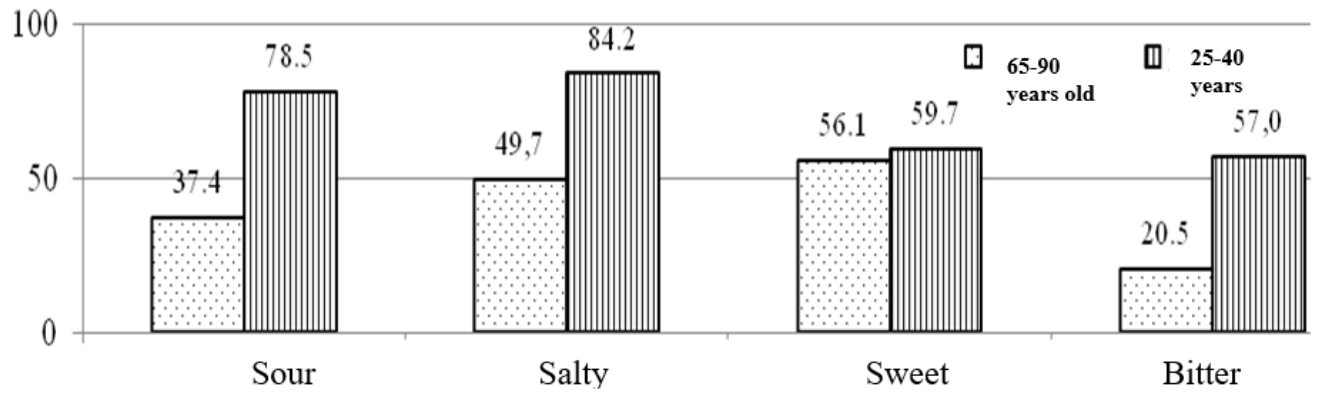

Fig. 1. Recognition by respondents of the main tastes in the age categories of 25-40 years and 65-90 years, $\%$

Recognition of the main tastes in the age group of $25-40$ years was $78.5 \%$ for sour, $84.2 \%$ for salty, $59.7 \%$ for sweet, and $57 \%$ for bitter; in the age group $65-90$ years old - for sour $-37.4 \%$, for salty $-49.7 \%$, for sweet $-56.1 \%$, for bitter $-20.5 \%$.

The data on the identification of sweet, salty, sour and bitter tastes in aggregate in different age categories are presented in Figure 2.

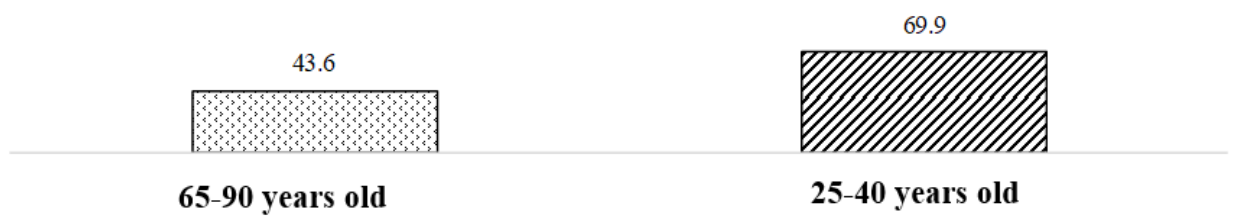

Fig. 2. Recognition of the main tastes in various age categories of $25-40$ years and $65-90$ years, $\%$

In order to find out the effect of diseases on the perception of tastes, respondents (65-90 years old) were offered a questionnaire for self-assessment of their health status. It was found that out of 383 respondents, 6 people had neoplasms, 15 people had diseases of the endocrine system (type 1, type 2 diabetes, thyroid disease), 86 people had diseases of the circulatory system (atherosclerosis, arterial hypertension of the 2nd and 3rd types, coronary heart disease - angina pectoris, myocardial infarction, etc.), 145 people - diseases of the digestive system (gastritis, duodenitis, gastric ulcer, etc.), 45 people - diseases of the genitourinary system (renal failure, etc.), 86 people - no diseases were detected, Figure 3.

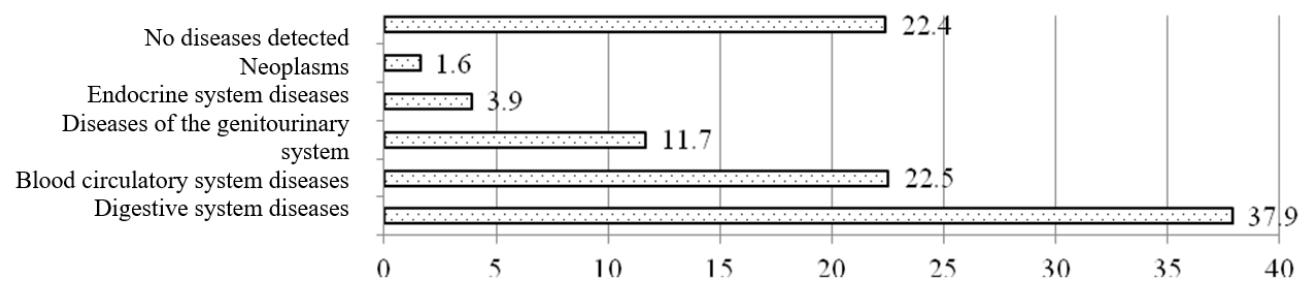

Fig. 3. Classes of diseases of respondents, $\%$

Digestive apparatus diseases occupy the first place - $37.9 \%$, then there are diseases of the circulatory system $-22.5 \%$, diseases of the genitourinary system $-11.7 \%$, diseases of the endocrine system $-3.9 \%$, neoplasms $-1.6 \%$.

Recognition of the main tastes by persons 65-90 years old with diseases in comparison with the average is presented in table 5 . 
Table 5. Recognition of the main tastes by persons $65-90$ years old with diseases in comparison with the average, $\%$

\begin{tabular}{|c|c|c|c|c|c|}
\hline Taste & $\begin{array}{c}\text { Average } \\
\text { indicator }\end{array}$ & $\begin{array}{c}\text { Gastritis, } \\
\text { duodenitis }\end{array}$ & $\begin{array}{l}\text { Hypertension, } \\
\text { myocardial infarction, } \\
\text { stroke }\end{array}$ & Renal failure & Diabetes \\
\hline Sour & $37,4 \pm 0,5$ & $29,3 \pm 0,5$ & $28,8 \pm 0,4$ & $29,5 \pm 0,5$ & $30,3 \pm 0,5$ \\
\hline Salty & $49,7 \pm 0,4$ & $33,8 \pm 0,4$ & $30,6 \pm 0,4$ & $32,3 \pm 0,4$ & $41,8 \pm 0,4$ \\
\hline Sweet & $56,1 \pm 4,0$ & $53,8 \pm 4,0$ & $47,2 \pm 4,9$ & $44,2 \pm 4,9$ & $40,3 \pm 4,0$ \\
\hline Bitter & $20,5 \pm 0,6$ & $17,6 \pm 0,6$ & $16,9 \pm 0,6$ & $16,0 \pm 0,6$ & $17,5 \pm 0,5$ \\
\hline
\end{tabular}

Recognition of the main tastes by people with diseases of the digestive system averaged $39.7 \%$, with diseases of the circulatory system $-37.7 \%$, with diseases of the endocrine system $-38.2 \%$, with diseases of the genitourinary system $-39.6 \%$.

Respondent recognition of odors is shown in Figure 4.

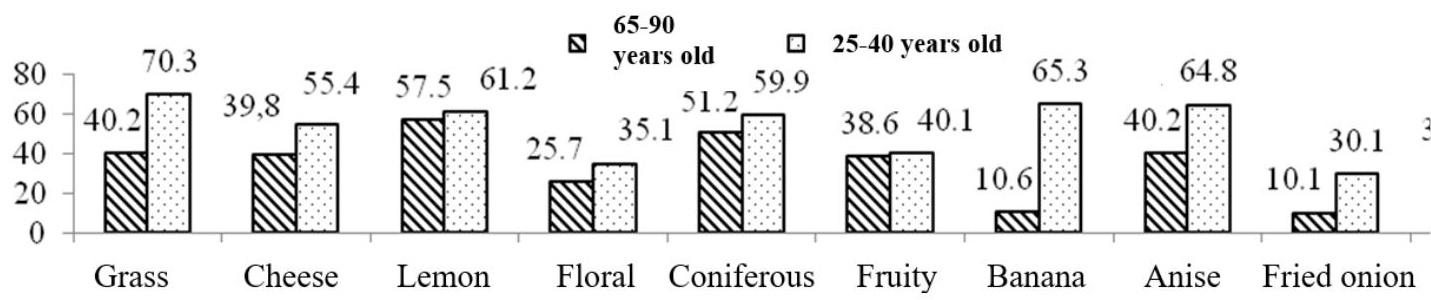

Fig. 4. Recognition of individual flavors by respondents, $\%$

The number of respondents who correctly identified all the samples proposed for recognition in aggregate by age groups is shown in Figure 5.

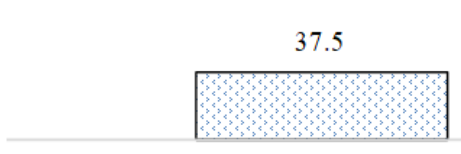

65-90 years old

Fig. 5. Respondents' recognition of odors, $\%$

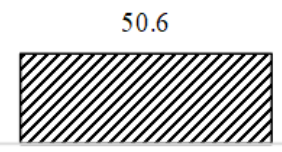

25-40 years old

From the data of the figure it follows that all odor samples proposed for identification were correctly identified by $37.5 \%$ of respondents aged $65-90$ years, $50.6 \%$ of respondents aged 25-40 years.

Results of a study of the effects of stevia extracts (stevioside and rebaudioside) on the solubility of curcumin. The turmeric powder alcohol solution was yellow. However, after centrifugation, removal of ethanol, subsequent dissolution in water and re-centrifugation, the supernant was clear and colorless. This fact confirms that curcumin extracted with ethanol has a low solubility in water. The alcohol solution with turmeric powder and stevioside was yellow. After centrifugation, removal of ethanol, subsequent dissolution in water and repeated centrifugation, the supernant was also yellow, indicating the presence of a water-soluble curcumin compound, figure.

The appearance of turmeric extracts before and after centrifugation, alcohol removal and dissolution in water, the appearance of the obtained extract is shown in Figure 6. 


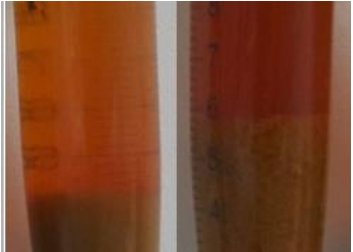

a

6

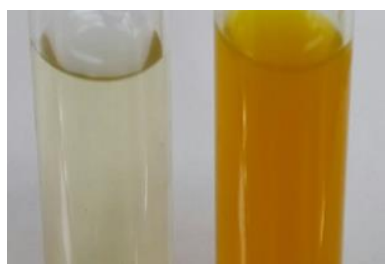

B

$\Gamma$

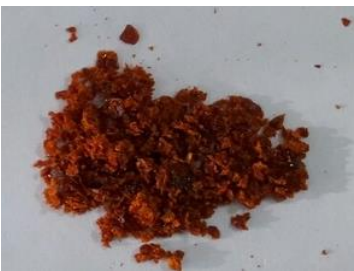

Д

$\mathbf{a}$ - curcuma powder with ethanol; $\boldsymbol{\sigma}$ - curcuma powder with ethanol and stevioside; $\mathbf{B}$ - curcumin aqueous solution obtained by ethanol extraction; $\mathbf{\Gamma}-$ an aqueous solution of the stevioside-curcumin complex obtained by extracting curcuma powder with ethanol with the addition of stevioside; $\boldsymbol{д}-$ the appearance of the stevioside-curcumin complex

Fig. 6. Appearance of curcuma extracts before and after centrifugation and dissolution in water

After receiving the complexes of stevioside-curcumin and rebaudioside-curcumin, their mass was determined. For example, the mass of the stevioside-curcumin complex obtained using $1 \mathrm{~g}$ of turmeric powder, $0.2 \mathrm{~g}$ of stevioside, is $0.11 \pm 0.02 \mathrm{~g}$, which is $9.17 \%$ of the amount of the starting ingredients. Then, the mass fraction of curcumin in the complexes was determined by photocolorimetry, table 8 .

Table 6. Evaluation of the solubilizing ability of stevia extracts

\begin{tabular}{|l|l|l|}
\hline \multirow{2}{*}{ Mass fraction of stevia extracts, $\%$} & \multicolumn{2}{|c|}{$\begin{array}{c}\text { The content of water-soluble curcumin in solutions (mg / } \\
\text { obtained with the addition of: }\end{array}$} \\
\cline { 2 - 3 } & stevioside & rebaudioside \\
\hline 0 & $0,38 \pm 0,02$ & $0,23 \pm 0,02$ \\
\hline 2 & $3,89 \pm 0,4$ & $3,72 \pm 0,4$ \\
\hline 4 & $9,67 \pm 0,4$ & $8,41 \pm 0,4$ \\
\hline 6 & $12,31 \pm 0,4$ & $10,52 \pm 0,4$ \\
\hline 8 & $14,33 \pm 0,4$ & $13,39 \pm 0,4$ \\
\hline 10 & $13,10 \pm 0,4$ & $11,70 \pm 0,4$ \\
\hline
\end{tabular}

As can be seen from the table, the maximum content of water-soluble curcumin was obtained in the stevioside-curcumin and rebaudioside-curcumin complexes at a concentration of $8 \%$, with a decrease or increase in this concentration, the amount of watersoluble curcumin begins to decrease. For further studies, the stevioside-curcumin complex will be used, since the rebaudioside-curcumin complex exhibits a lower curcumin content $14.33 \pm 0.3$ and $13.39 \pm 0.3 \mathrm{mg} / \mathrm{ml}$, accordingly.

Results of modeling gerontological drinks. At an earlier stage of the study, the authors developed gerontological whey drinks [24] containing the following mass fractions of components: cottage cheese whey $-60.0 \%$, sugar syrup $-7.0 \%$, dry green tea extract $0.4 \%$, juice sea buckthorn $-10.0 \%$, carrot juice $-5.0 \%$, cranberry juice $-1.0 \%$, apple pectin $-0.02 \%$, vitamin premix $-0.025 \%$, Pronadisa yeast extract $-0.01 \%$, water - up to $100 \%$.

Since milk curd whey contains about $0.9 \mathrm{~g} / 100 \mathrm{~g}$ protein, to increase the intensity and harmonize the flavor of drinks, yeast extract was added to the formulations, which is rich in sweet amino acids - proline, glycine and alanine. It contains glutamic acid, which in combination with whey proteins enhances the taste of drinks, making it brighter. In addition, to improve the organoleptic properties of drinks, apple pectin is present in the formulations, which forms the body of the drink, prolongs the aftertaste, gives the taste richness, depth, moreover, the taste is softened (the drink turns out to be less acidic), the berry, vegetable and extract tastes are combined, thanks to which, they are felt together. 
In order to develop drinks with a low glycemic index, they decided to add the obtained stevioside-curcumin complex to the drinks formulations instead of sugar. For this, its sweetness was determined by comparison with the sweetness of sucrose solutions in various concentrations. The complex was found to be 100 times sweeter than sugar. Based on this, a complex was added to the composition of previously developed beverage recipes based on the calculated sweetness and daily requirement for curcumin $-0.017 \%$, which amounted to $15.0 \%$ of the adequate level of consumption $(50 \mathrm{mg})$ established by TS TS $021 / 2011$. Then, the profile of the sweetness of drinks with the addition of the steviosidecurcumin complex was compared with the profiles of the sweetness of drinks prepared with added sugar, aspartame. The results are presented in Figure 7.

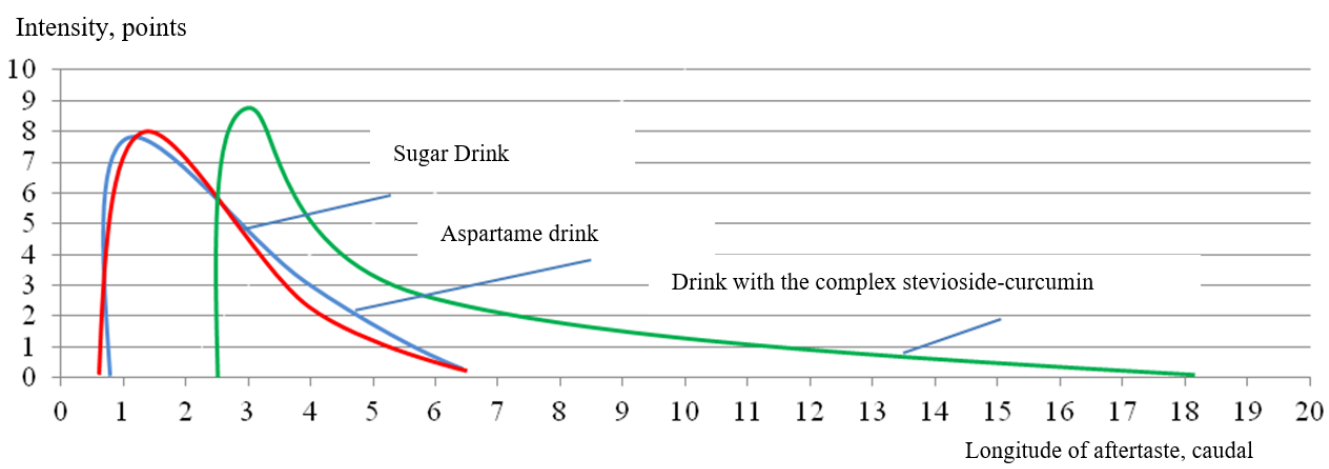

Fig. 7. Sugar, aspartame, stevioside-curcumin complex sweetness profiles

From the data of the figure it follows that the intensity of the taste of drinks with sugar and aspartame is 7 points, the longitude of the aftertaste is 5.5 caudal; for drinks with the addition of the stevioside-curcumin complex, the indicators are 8.5 points and 15 caudal, respectively. The intensity of the taste of the drink with the complex is $15 \%$ more, the longitude of the aftertaste is 2.73 times more. However, the sweetness of drinks with the addition of the complex begins to be felt not from the first second, as in drinks with sugar and aspartame, but only after 2.5 seconds. This reduces the organoleptic indicators of the quality of the drinks. Therefore, a mixture of aspartame with the stevioside-curcumin complex was added to the composition of the drinks to achieve the desired sweetness profile, Figure 8.

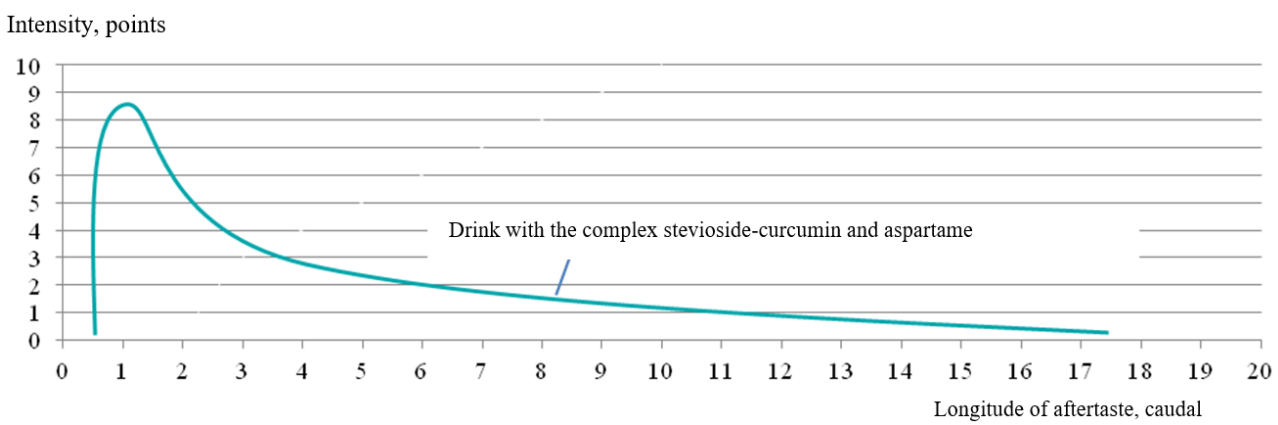

Fig. 7. The profile of the sweetness of the drink with the addition of the stevioside-curcumin complex and aspartame

Aspartame was added in an amount of $0.01 \%$. As a result, the resulting drink in the profile of sweetness is close to that of sugar, the aftertaste longitude is 3 times greater and 
amounts to 16.5 caudal, the taste intensity is $15 \%$ higher, the sweetness is felt from the first second. Curcumin, with a sharp taste, activates taste buds.

Then, consumer tastings were conducted to compare the flavor intensity of the developed beverage samples with the competitor's drink, Figure 8.

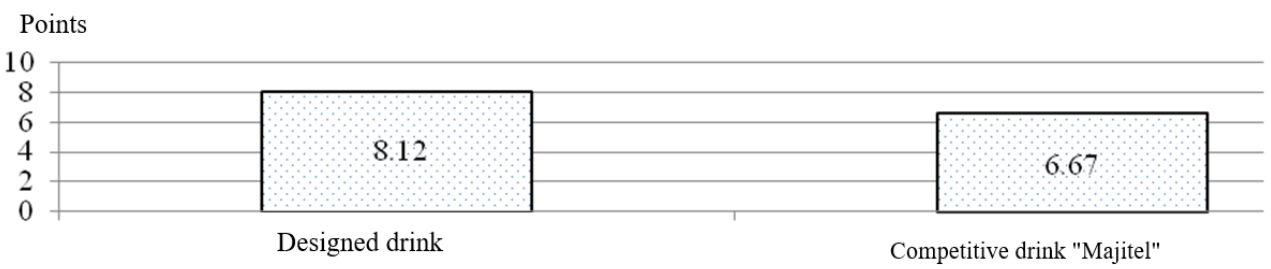

Fig. 8. The flavor intensity of the developed samples of drinks and a competitor beverage, points

From the data of the figure it follows that the intensity of the developed drink, according to consumers, amounted to 8.12 points, a competitor drink - 6.67 points. This circumstance confirms the hypothesis of the study about the possibility of obtaining gerontological drinks with a more saturated taste and intense aroma for the elderly people.

Increased functional properties of the drinks were ensured by introducing into the composition of the complex stevioside-curcumin and components belonging to the class of functional food ingredients that have an antioxidant effect: vitamin $\mathrm{C}$ and $\mathrm{E}$, carotenoids, flavonoids in accordance with GOST R 54059-2010 [25]. According to one of the classifications [26], curcumin also belongs to the category of functional food ingredients of plant origin (natural food colors) and is used in the production of dairy products. Figure 9 shows the content of components that have an antioxidant effect in a portion of the drink $(250 \mathrm{ml})$.

Satisfaction with daily requirements, $\%$

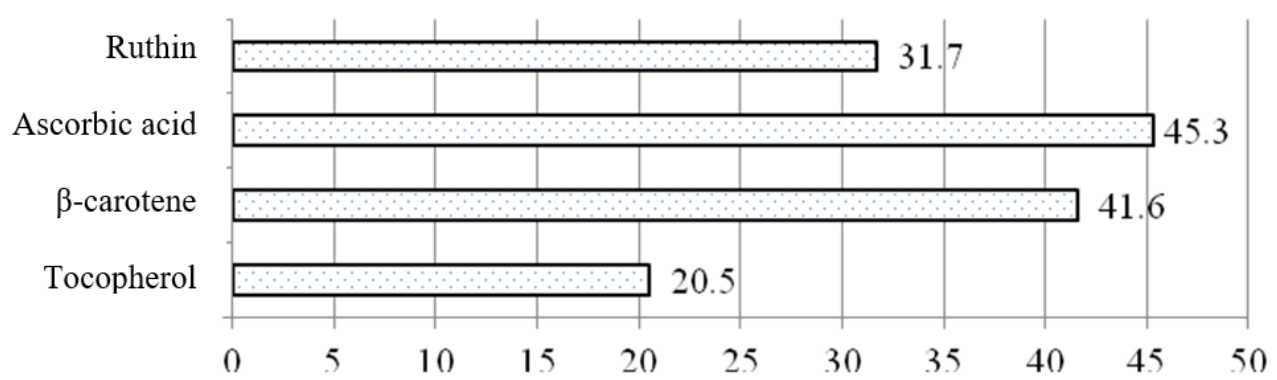

Fig. 9. The content of components that have an antioxidant effect in a serving of drink, $\%$

Drinks contain from 20.5 to $45.3 \%$ micronutrients, which meets the requirements of GOST R 52349-2005. Then, the antioxidant activity of the developed samples of drinks and a competitor beverage was measured by potentiometry [27]. The unit of measurement of antioxidant activity in this method is mmol-eq / 1, the results are presented in Figure 10.

Antioxidant activity, mmol-equiv / 1

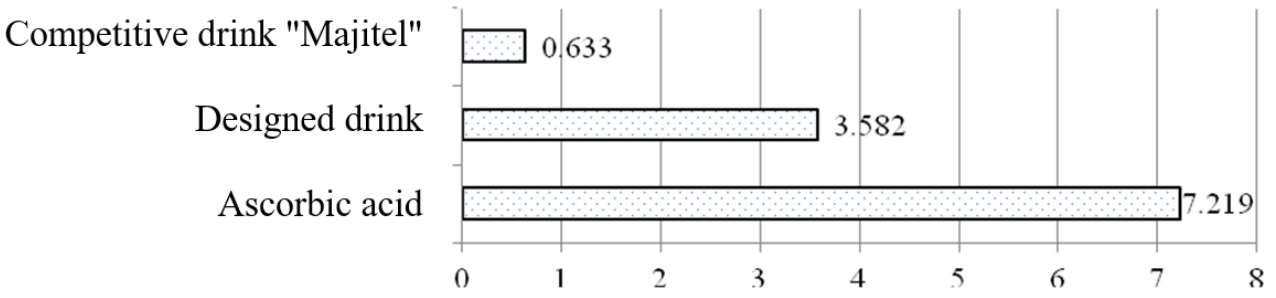


Fig. 10. Antioxidant activity of samples, mmol-equiv / 1

An aqueous solution of ascorbic acid - $90 \mathrm{mg} / 100 \mathrm{ml}$, which is $100 \%$ of the recommended daily intake, was used as a standard for antioxidant activity. The antioxidant activity of the developed drink was $3,582 \pm 0,04$, the competitor drink $0,633 \pm 0,005$ mmolequiv / 1 , which is $49.6 \%$ and $8.8 \%$ of the antioxidant activity of ascorbic acid, accodingly.

\section{Discussion}

The results of the study of taste sensitivity in the age group of 25-40 years were compared with the results of the study in the age group of 65-90 years. The recognition of tastes in individuals in the age group $65-90$ years is worse: for salty - by $41.0 \%$, for sour - by $52.3 \%$, for bitter - by $64.0 \%$, for sweet, the indicators are lower by $6.0 \%$. Persons aged $65-90$ years recognized basic tastes worse by $37.6 \%$ in total. The obtained data are correlated with literature data and confirm the tendency for a decrease in sensory sensitivity with age towards (salty, bitter, sour tastes) with minimal deterioration in the ability to recognize sweet taste.

The indicators obtained in the study of the taste sensitivity of people 65-90 years old with diseases were compared with those of healthy people. People with diseases of the digestive system recognized the main tastes worse by $22.9 \%$, with diseases of the circulatory system - by $26.8 \%$, with diseases of the endocrine system - by $25.2 \%$, urogenital system - by $26.1 \%$. People suffering from diseases of the digestive system, diabetes mellitus, noted a decrease in taste sensitivity for all types of tastes, which is explained by dystrophic changes in the epithelium of the tongue, taste buds, and the occurrence of dryness in the oral cavity. These changes occurred against the background of a long and difficult course of the pathology of internal organs and make it difficult to recognize tastes. Therefore, a decreased perception of basic tastes by elderly people can be associated with various diseases.

Odor recognition by individuals aged $65-90$ years is $25.9 \%$ worse compared to persons aged 25-40 years. For respondents aged 65-90, the main difficulty was the recognition of aromas: floral, fruit, banana, fried onions; 25-40 years old - floral, fruit, fried onions. The simplest identifiers were odors for a group of 65-90 years old: lemon, pine, anise, almonds; for a group of 25-40 years old: grass, cheese, lemon, pine, banana (strawberry), anise, almonds. Odor recognition in individuals aged $65-90$ years is $25.9 \%$ worse compared to persons aged 25-40 years. The results of the study are interconnected, primarily with impaired short-term memory, associative perception.

When curcumin was extracted with ethanol with the addition of $8 \%$ stevia extracts, the maximum yield of the water-soluble curcumin compound for stevioside and rebaudioside was observed $-14.33 \pm 0.3$ and $13.39 \pm 0.3 \mathrm{mg} / \mathrm{ml}$, respectively. For stevioside, the indicator is $6.56 \%$ higher. At higher or lower concentrations of stevia extracts, the yield decreased. When curcumin was extracted with ethanol or water without the addition of stevia extracts, water-soluble curcumin was not detected or was found in insignificant amounts for stevioside and rebaudioside $-0.38 \pm 0.02$ and $0.23 \pm 0.02 \mathrm{mg} / \mathrm{ml}$, accordingly. The mechanism of interaction of turmeric compounds with stevia extracts has not yet been established.

During the simulation of beverage recipes, it was found that in order to achieve high organoleptic characteristics, the complex must be added in an amount of $0.017 \%$, which is $15.0 \%$ of the adequate level of consumption. With an increase in the mass fraction of the complex in the drink, the smell of curcumin became prevailing, the taste was excessively sharp and sweet, and the harmony of flavor was disturbed. The saturation of the taste of the drink with the addition of the stevioside-curcumin complex is $15 \%$ higher, which, apparently, is explained by the ability of stevia extracts not only to enhance sweetness, but 
also to increase the intensity of the natural aroma of fruits and fruits. Curcumin, with a sharp taste, activates taste buds, which also enhances the taste. Additionally, an increase in the intensity of taste, long aftertaste and harmonization of the flavor of the drinks was achieved by adding a yeast extract and apple pectin. As a result of consumer tastings, it was found that the flavor intensity of the developed samples of drinks is $14.5 \%$ higher compared to a competitor. Drinks due to the content of components that have an antioxidant effect, have high antioxidant activity. The antioxidant activity of the developed drink is 5.7 times greater than that of a competitor's drink.

\section{Conclusions}

During the study, quantitative values were obtained in relation to the taste and olfactory sensitivity of individuals in two age categories - 25-40 years old and 65 years and older: it was found that the recognition of tastes in individuals in the age category 65-90 years is worse for salty - by $42.0 \%$, for sour - by $61.0 \%$, for bitter - by $65.6 \%$, for sweet indicators are lower by $19.2 \%$. People with diseases of the digestive system recognize basic tastes worse by $22.9 \%$, with diseases of the circulatory system - by $26.8 \%$, with diseases of the endocrine system - by $25.2 \%$, urogenital system - by $26.1 \%$. Odor recognition by individuals aged $65-90$ years is $25.9 \%$ worse compared to persons aged $25-40$ years. The effect of stevia extracts (stevioside and rebaudioside) on curcumin solubility was studied: when curcumin was extracted with ethanol with the addition of $8 \%$ stevia extracts, the maximum yield of curcumin for stevioside and rebaudioside was $34.4 \pm 0.4$ and $32.3 \pm 0.3$ $\mathrm{mg} / \mathrm{ml}$, accordingly. For stevioside, the indicator is $6.1 \%$ higher. It was found that the obtained stevioside-curcumin complex, containing water-soluble curcumin in bioavailable form, is 100 times sweeter than sugar. The gerontological drinks with the addition of the steviosis-curcumin complex in the amount of $0.017 \%$, which amounted to $15.0 \%$ of the adequate level of curcumin consumption ( $50 \mathrm{mg}$ ), established by TP TS $021 / 2011$, were modeled. The resulting drink in the profile of sweetness is close to that of sugar, the aftertaste longitude is 3 times greater and amounts to 16.5 caudal, the taste intensity is $15 \%$ higher, the sweetness is felt from the first second. The enhanced functional properties of the drinks were ensured by introducing the stevioside-curcumin complex and components belonging to the class of functional food ingredients that have an antioxidant effect. The antioxidant activity of the developed drink is 5.7 times greater than that of a competitor's drink.

\section{References}

1. G. Casamatta, L. Batte, Handbook of the Economics of Population Aging, 1, 381-444 (2016)

2. J. Gopal, M. Muthu, and S.C. Chun, RSC Advances, 5, 48391-48398 (2015)

3. The elderly population of Russia: problems. http://ac.gov.ru/files/publication7a/8485.pdf.

4. Incidence of the population over working age in Russia in 2017. https://www.rosminzdrav.ru.

5. Dr. John, R. Beard, A. Officer, The Lancet, 387, 10033, 2145-2154 (2016).

6. R. L. Doty. and V. Kamath, Front Psychol, The influences of age on olfaction: a review, 4, 6-20 (2014)

7. L. Methven, V. J. Allen, C. A. Withers, Proceedings of the Nutrition Society, Ageing and taste, 71 (4), 556-565 (2012) 
8. K. Solemdal, T. L. Sandvik, Gerodontology, 31 (1), 42-48 (2014)

9. J.D. Wessler, S. L. Hummel, and M. S. Maurer, Progress in Cardiovascular Diseases, 57 (2), 160-167 (2014)

10. J. Chen and A. Rosenthal, Sensory Analysis, Consumer Requirements and Preferences Modifying Food Texture. V.2 (UK: Woodhead Publ, 2015)

11. N.S. Bukreev, Scientific notes of the Russian State Social University, Modern research in the field of smell and smell Modern research in the field of smell and smell, 135, 1421 (2016)

12. D. Lafreniere, N. Mann, Otolaryngologic Clinics of North America, 1 (387), 123-131 (2009)

13. D. Ellison, D. White, Aging Population, 1 (50), 185-213 (2015)

14. C.V. Chandrasekaran, K. Sundarajan, J.R. Edwin, Pharmacognosy Research, 5 (2), 71 79 (2013)

15. E.I. Reshetnik, E.A. Utochkina, Food and Raw Materials, 1, 88-94 (2013)

16. V.C. Sezgin, O. Bayraktar, Production of curcumin and piperine loaded doublelayered biopolymer based nano delivery systems by using electrospray / coating method. Patent US, no. EP 3142702 B1 (2018)

17. R. Jäger, R, Lowery, A. Calvanese, Nutrition Journal, 13, 4-8 (2014)

18. J-L. Ji, X-F. Huang, H-L. Zhu, Anti-Cancer Agents in Medicinal Chemistry, 3 (12), 210-218 (2012)

19. F. Zhang, G.Y. Koh, D.P. Jeansonne, Journal of Pharmaceutical Sciences, 7 (100), 2778-2789 (2011)

20. H.G. Cave, Improved complexes and compositions containing curcumin. Patent US, no. WO 2013176555 A1 US, (2013)

21. D. Benam, Curcumin Solubilizate Curcumin Solubilizate / Pat. RU 2615815 (2017)

22. O.A. Legonkova, A.I. Korotaeva, R.P. Terekhova, Pat. RU 2665378 A method of obtaining a biologically active composite based on nanocrystalline cerium dioxide and curcumin (2018)

23. N. Nguyen, J-B Si, C. Kang, Food Chemistry, 214, 366-373 (2017)

24. N.V. Zavorokhina, Yu.I. Bogomazova, A.V. Tarasov, Food Industry, 8, 70-74 (2018)

25. GOST R 54059-2010. Functional food products. Physiologically Functional Ingredients Functional food products. Functional Food Ingredients.: GOST R540592010. - Vved. 01-01-2011. - M .: Standartinform. (2010)

26. M.N. Shkolnikova, E.V. Averyanova, Bulletin of the Krasnoyarsk State Agrarian University, 9, 85-92 (2017)

27. H.Z. Brainina, A.V. Ivanova, E.N. Sharafutdinova, Talanta, 1 (71), 13-18 (2007) 